\title{
Carpometacarpal Hemiarthroplasty
}

\author{
Gerson B Florez ${ }^{* 1}$ and Francisco Rubio ${ }^{2}$ \\ ${ }^{1}$ Orthopaedic Hand and Upper Extremity Surgeon, St. Clair Orthopedic Associates, St. Clair Hospital, USA \\ ${ }^{2}$ Orthopaedic Hand and Upper Extremity Surgeon, Miami Hand \& Upper Extremity Institute, USA
}

Submission: August 23, 2017; Published: August 29, 2017

*Corresponding author: Gerson B Florez, Orthopaedic Hand and Upper Extremity Surgeon, St. Clair Orthopedic Associates, St. Clair Hospital, Pittsburgh, PA, USA, Email: gerson.florez@stclair.org

\begin{abstract}
Thumb carpometacarpal (CMC) arthritis is a common condition that can be painful and debilitating. The versatility of the first carpometacarpal joint and the requirements of daily activities with pinch and grip strength results in forces across the first CMC joint that may increase up to twelve times the applied force on the thumb pad, [1] ultimately resulting in degenerative arthritis. While many surgical techniques have been described to treat this problem, the concept of hemiarthroplasty remains attractive because it prevents metacarpal shortening, maintains pinch and grip strength, [2-17] decrease pain and stiffness, and allows for earlier recovery of strength and function.
\end{abstract}

Our experience with Stablyx® Arthroplasty System (Skeletal Dynamics, LLC, Miami, FL), a hemiarthroplasty for the carpometacarpal joint, demonstrates favorable results in 120 procedures. In a 24-month period, 12 patients have been followed to assess pain, function, strength, range of motion and trapezial wear. Pain was a $0.06 \pm 0.18$ at rest, and $0.34 \pm 0.98$ during active motion on a 10-point visual analog scale (VAS). The QuickDASH score was a $2.37 \pm 0.86$, where 0 is no disability and 100 is total disability. The patients' grip strength at 24 months was $106 \% \pm 26 \%$ and pinch strength was $123 \% \pm 62 \%$ of the contralateral hand. Patients achieved full range of motion by 24 months. Trapezial wear at 24 months, analyzed using a PACSgreen software, demonstrated a trapezial height that was $99.4 \% \pm 5.4 \%$ of the trapezial height measured at the first postoperative visit (5-7 days post-surgery).

Since the hemiarthroplasty retains the trapezium it is vital that surgical dissection preserves the dorsal perforating branches that provides blood supply to the trapezium. A modification to the surgical dorsal approach of the carpometacarpal joint is described to minimize osteonecrosis of the trapezium using a ulnarly-based capsulotomy distal to the trapezium. Successfully treated patients with hemiarthroplasty in one limb versus a ligament reconstruction tendon interposition (LRTI) in the contralateral limb have expressed that they would undergo the hemiarthroplasty as their first choice between the two procedures.Since the current solution of hemiarthroplasty for thumb CMC arthritis demonstrates early patient satisfaction and preserves techniques in the event of implant failure, we would recommend the use of hemiarthroplasty as an alternative to ligament reconstruction tendon interposition in patients with Eaton stage II or Eaton stage III arthritis with good trapezium bone stock.

Keywords: CMC hemiarthroplasty; Implant; Thumb osteoarthritis

\section{Introduction}

The opposable thumb, an integral part of our daily hand function, has evolved across species and has resulted in the functions of prehension, circumduction, and opposition. These functions are a combination of planar movements across the first carpometacarpal (CMC) joint that enables adduction-abduction, flexion-extension, and axial rotation [1]. The versatility of the first CMC joint in multiple planes is attributed to the lack of bony constraints and the saddle articulation relying on the capsuloligamentous structures to provide stability. During key pinch the forces across the first CMC joint may increase up to twelve times the applied force on the thumb pad [2]. The combination of the increased compressive reactive forces with joint laxity results in shear stresses that degrade the cartilage over time and lead to degenerative osteoarthritis and pain.
There are over 2000 hand surgery specialists in the United States [3] within these hand clinics 5\%-33\% of patients are seen for thumb carpometacarpal arthritis and its symptoms, including pain, thumb fatigue, and loss of prehensile strength [1]. Many different surgical techniques have been devised over the past 60 years in efforts to provide patients with pain relief and early return to work. Of the various techniques, ligament reconstruction and tendon interposition (LRTI) has been the mainstay treatment among most surgeons [1-5].

Prosthetic interposition implants were reported in the 1980s with the advancement of silicone spacers and demonstrated promising results initially, with pain relief and improved function without loss of thumb length.75\% of patients with silicone implants reported satisfaction with their outcome 
after silicone arthroplasty [7]. Long-term results demonstrated silicone synovitis and adjacent bone erosion with an end result of repeat joint subluxation, instability and high rate of revision. Pellegrini and Burton reviewed a series of 32 silicone thumb interposition arthroplasties demonstrating subluxation and loss of height, resulting in $16 \%$ of the case series undergoing revision (5 patients) [8].

In 1984, in reaction to the poor long-term results of silicone elastomer implants, surgeons explored titanium and pyrocarbon hemiprostheses. By 1997, Swanson et al. published a 10-year series of 105 titanium hemiarthroplasties in a group of 92 patients. Swanson demonstrated successful outcome in 89 of the 92 patients, based on improved pain relief, better motion, and $33 \%$ and $64 \%$ improvement in tip pinch and key pinch strength, respectively [9]. Toreproduce Swanson's 1997 success with the titanium convex condylar hemiarthroplasty for CMC arthritis, Phaltankar and Mangussen performed 19 arthroplasties in 18 patients using the Swanson implant. They reported on 18 of the 19 arthroplasties demonstrating 1 dislocation requiring revision, 15 of 18 patients with good pain relief (mean VAS for pain being 3 on a scale of $0-10$, where $10=$ intolerable), and 18 patients with excellent hand function (mean VAS for hand function of 2 on a scale of $0-6=$ normal hand function and $10=$ no function).They also noted a high rate of radiographic loosening on the metacarpal side in 5 of 18 joints and trapezial wear in 10 of 18 joints. However, radiographic loosening or trapezial wear did not correlate with worse hand function or increased pain [10].

Pyrocarbon, a synthetic material that has a modulus of elasticity similar to cortical bone, [11] was developed in the 1960s and ultimately applied to the field of CMC joint replacement to address the problem of bone resorption seen with silicone implants. The hopes were that the material would increase long-term survivability because of its biological compatibility and to enhance biological fixation by decreasing the stresses at the bone prosthesis interface. Good short-term and longterm results of pyrocarbon metacarpophalangeal (MCP) joint implants, $[12,13]$ influenced Martinez de Aragon et al to trial the proximal component of MCP implants as a hemiarthroplasty for the trapeziometacarpal joint in 49 patients [14].

As the implant was not designed specifically for the carpometacarpal joint, Martinez de Aragon's study found a $16 \%$ failure rate. Thirteen of the fifteen cases requiring revision were due to dislocation of the implant. In 2012, Pritchett followed up with a series of 159 he miarthroplasties of the thumb CMC using a porous coated titanium stem designed specifically for the CMC joint. The implant had a unique modular head component that allowed for head diameter adjustments and length adjustments for tensioning. The overall outcomes demonstrated excellent to good results in 139 arthroplasties without any dislocations. One hundred and thirty-five thumbs described occasional or no pain and 138 thumbs having good to excellent functional improvement. Eleven patients had previously undergone a ligament reconstruction and tendon interposition in the contralateral thumb and preferred the implant arthroplasty results [15].

With new technological advancements in manufacturing, the hemiarthroplasty prosthesis for thumb carpometacarpal joint arthritis is being revisited using a mono-block, cobalt chrome, saddle shaped implant. The implant is intended for uncemented, press fit use to take advantage of the stem's titanium plasma spray coating for increased biological fixation. The advantages of the new design include a highly polished anatomic replication of the articular surface of the thumb metacarpal base, a keel built into the stem to set the positioning and improve rotational stability, optional suture holes to augment stability for cases with severe laxity, and manufacturing techniques to minimize joint reaction forces and alleviate edge loading.

To address the dorsal subluxation of first generation implants, the new design has a longer palmar lip that sits volar to the trapezium serving a mechanical stop to dorsal subluxation. Even though prior studies of the first-generation implants demonstrated radiographic osteolysis around alloyed implants without functional impairment, $[9,10]$ the features of the new generation add the potential for bony in growth and supportive joint reaction forces. The concept of hemiarthroplasty remains attractive because it prevents metacarpal shortening which has been found to decrease pinch and grip strength, $[16,17]$ decrease pain and stiffness, and allows for earlier recovery of strength as seen in previous implant arthroplasty literature [7,9,10,14-16].

A disadvantage of a Cobalt Chrome implant for CMC arthroplasty is that the implant has a higher modulus of elasticity in comparison to titanium and cortical bone. The modulus mismatch results in higher stresses at the bone-implant interface leading to micromotion and possible ingrowth failure $[11,18]$. The implant cannot directly address scaphotrapeziotrapezoid (STT) arthritis; however, if STT joint arthritis exists it may be addressed by excising the distal pole of the scaphoid during the implant procedure or with a traditional ligament reconstruction tendon interposition (LRTI).

After failed conservative treatment of carpometacarpal arthritis, indications for surgery include persistent pain with activities of daily living, decreased function, and instability. The added indications for hemiarthroplasty would be younger patients in their 5 th and 6 th decade of life without concomitant scaphotrapeziotrapezoid arthritis, have maintenance of the trapezial height, and have an increased demand for strength during physical activity. Preserving the height of the first metacarpal has correlated with increased key and pitch strength $[16,17]$ thus hemiarthroplasty with a prosthesis would preserve length and serve young patients who are still in the work force performing manual tasks or are active participants of routine sporting activities. Exclusion criteria for hemiarthroplasty would 
include infection, pantrapezial and scaphotrapeziotrapezoid arthritis, or poor bone stock.

\section{Technique}

It is recommended that a patient receive a regional anesthetic block for pain relief and is placed under monitored care anesthesia or general anesthesia. The patient may remain on the hospital gurney during the procedure. While in the supine position the affected arm is place on a roll away padded hand table. Fluoroscopy is required during the case to evaluate the trial prosthesis prior to implanting the final components. The arm is exsanguinated with an Esmarch and a tourniquet is placed at the level of the Humerus at $200 \mathrm{mmHg}$.

a) A standard dorsal, longitudinal $4 \mathrm{~cm}$ incision is centered over the base of the metacarpal and CMC joint running adjacent to the extensor pollicis brevis (EPB) tendon (Figure 1).

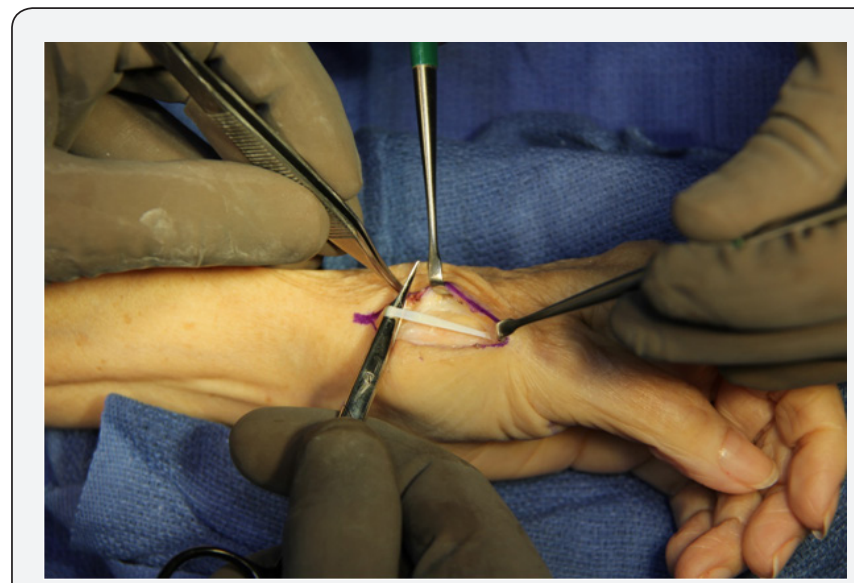

Figure 1: Dorsal incision with EPB identified.

b) Dissect the subcutaneous tissue and identify the dorsal radial sensory branch and EPB tendon. Retract the EPB tendon ulnarly to create the window between the Abductor Pollicis Longus (APL) tendon and the EPB tendon.

c) Identify and protect the radial artery within this window.

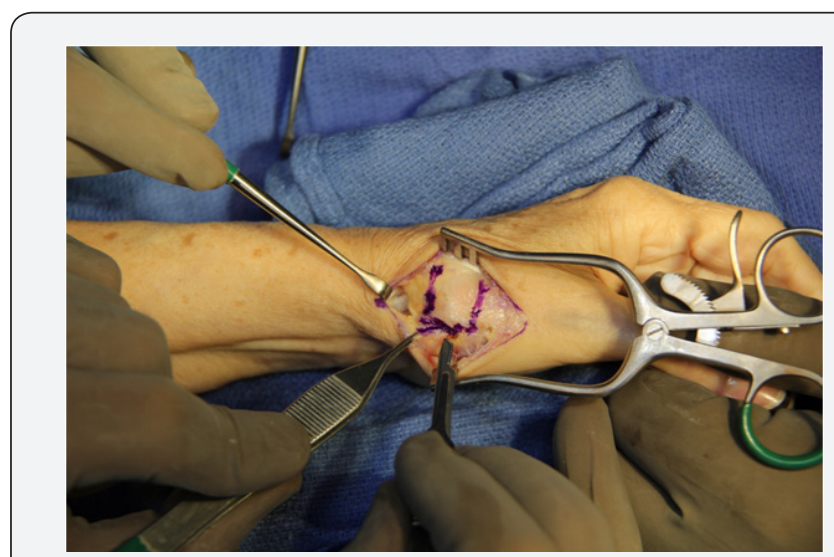

Figure 2: Dorsal capsule with ulnar based flap marked with purple ink. d) Identify the metacarpotrapezial joint and create an ulnar based capsular flap that is centered over the joint. Recall that the main blood supply to the trapezium is from dorsal bone perforators. Since this procedure retains the trapezium, all efforts should be made to preserve the vascularity of the trapezium to prevent osteonecrosis. Longitudinal capsulotomies should not be performed as this is more likely to devitalize the trapezium (Figure 2).

e) Using a bone holding forceps to hold the thumb metacarpal, partially release the APL tendon to allow easier access to the base of the metacarpal. Draw the longitudinal axis of the dorsal metacarpal with a marking pen or electrocautery bovie. Make a perpendicular osteotomy with an oscillating saw $6 \mathrm{~mm}$ distal to the joint surface (Figure 3).

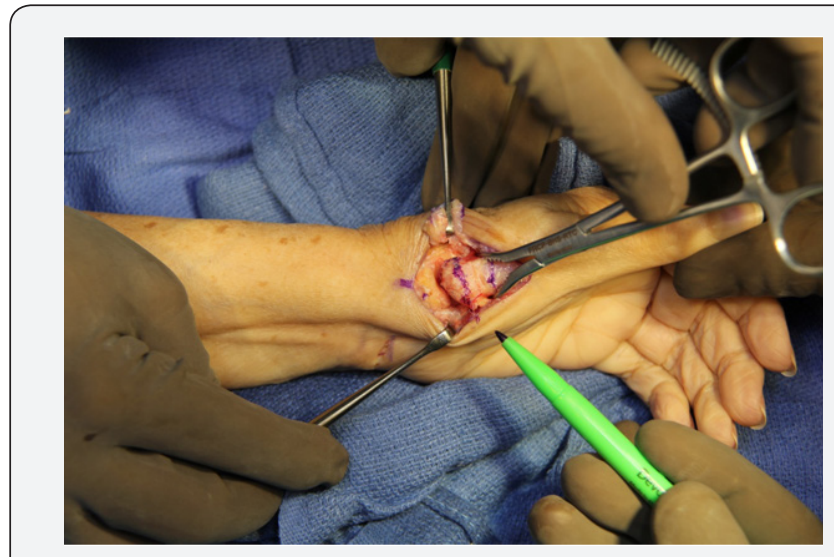

Figure 3: Bone markings identifying the osteotomy site for the metacarpal.

f) Expose the joint to remove excess synovium and gain access to the volar capsule and remove volar metacarpal osteophytes. Turn your attention to the trapezium and remove dorsal osteophytes without damaging the cartilaginous face of the trapezium or injuring the blood supply (Figure 4).

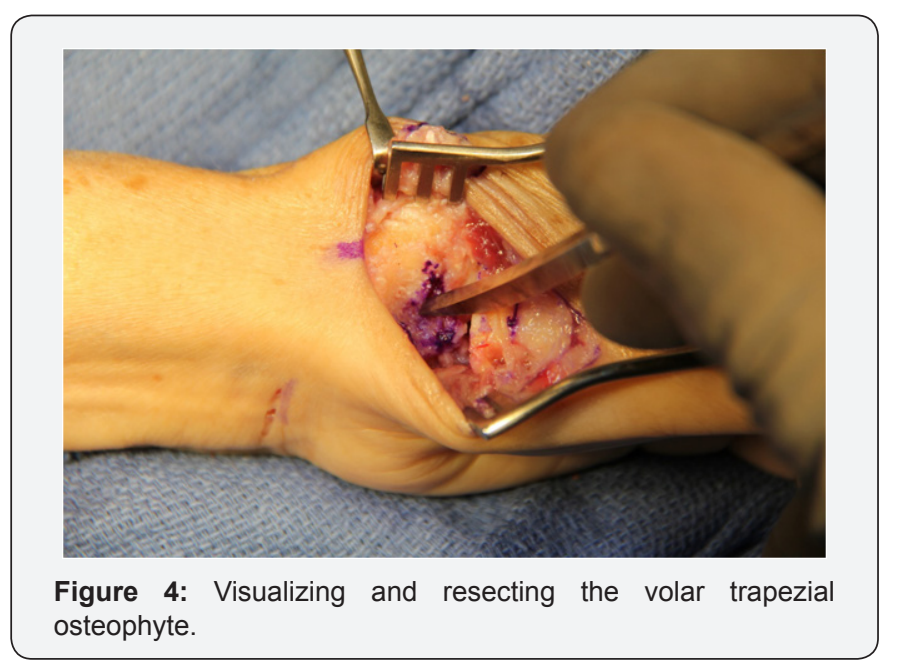

g) Use a capsular elevator to tease off the volar capsule from the trapezium taking care not to injure the Flexor Carpi Radialis (FCR) tendon exiting from the FCR tunnel. This will help 
you visualize the volar trapezial osteophyte. Mark the osteophyte at the cartilage margin and remove using a curved osteotome. Do not remove healthy palmar bone, as it is essential for supporting the implant. Now mobilize the FCR from the trapezium to allow space for the volar flange of the implant.

h) Insert the trapezial rasp between the FCR tendon and the volar lip of the trapezium and rasp from proximal to distal to remove any remaining volar osteophytes. Insert the trapezial contouring tool and rasp the central palmar aspect of the trapezium in an oscillating fashion from radial to ulnar and proximal to distal to create a saddle shaped contour.

i) Size the trapezium with the provided sizing instrument. The correct size will allow the sizing tool to translate $1 \mathrm{~mm}$ in the volar-dorsal direction and can sweep from radial to dorsal across the entire width of the trapezium. If the sizer cannot be swept from radial to ulnar, remove any remaining trapezial osteophytes (Figure 5).

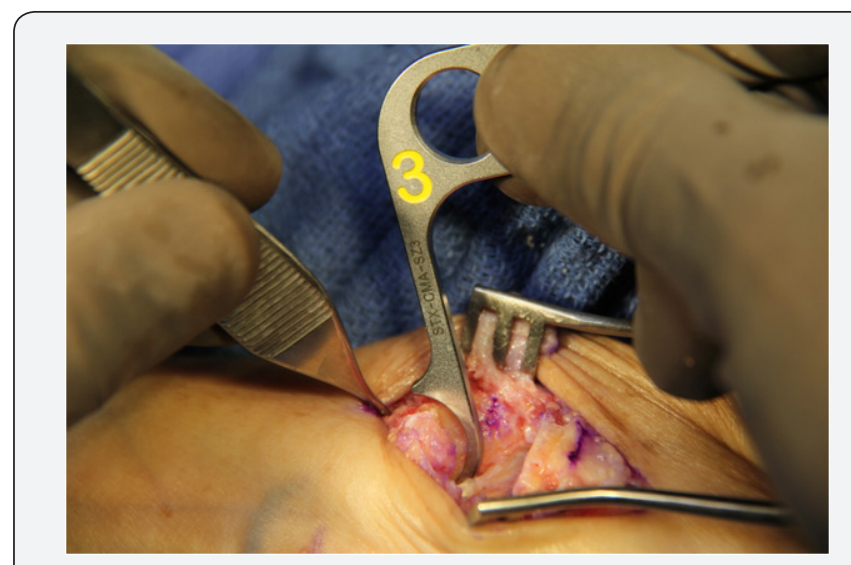

Figure 5: Measuring the trapezium size.

j) Attention is turned to the base of the thumb metacarpal. Insert the straight starting awl down the medullar canal. Once the awl is within the metacarpal take fluoroscopic images with a mini C-arm in PA and Lateral planes to confirm that the awl is central within the medullary canal.

k) Prepare the metacarpal by sequentially rasping the medullary canal, with the provided metacarpal rasps, up to the corresponding size measured on the trapezium. If the metacarpal will not allow the corresponding trapezial size, turn back to the trapezium and continue removing dorsal bone (preserving as much palmar bone as possible) to obtain a matching size between the metacarpal and the trapezium.

l) To establish metacarpal rotation, place the trial implant, that is one size smaller than was measured to allow easy rotation, into the medullary canal of the metacarpal base. Oppose the thumb to the small metacarpal head and mark the location of the tab on the metacarpal with a bovie. Now place the thumb into full radial abduction and mark the location of the tab on the metacarpal with a bovie. Both marks usually coincide, if not, the midpoint of the two markings will be the correct position of the final implant.

m) Replace the undersized trial with the correct trial size using the defined rotational alignment. Reduce the trial implant to the trapezium, being careful not to injure the articular cartilage. A technical pearl to protecting the cartilage is to place the capsular elevator provided over the articular surface of the trapezium which can then be used as a sled to help reduce the implant. Place the thumb into full opposition and confirm that the trial implant does not hinge. If hinging occurs the volar lip may be impinging on a remaining osteophyte (Figure 6).

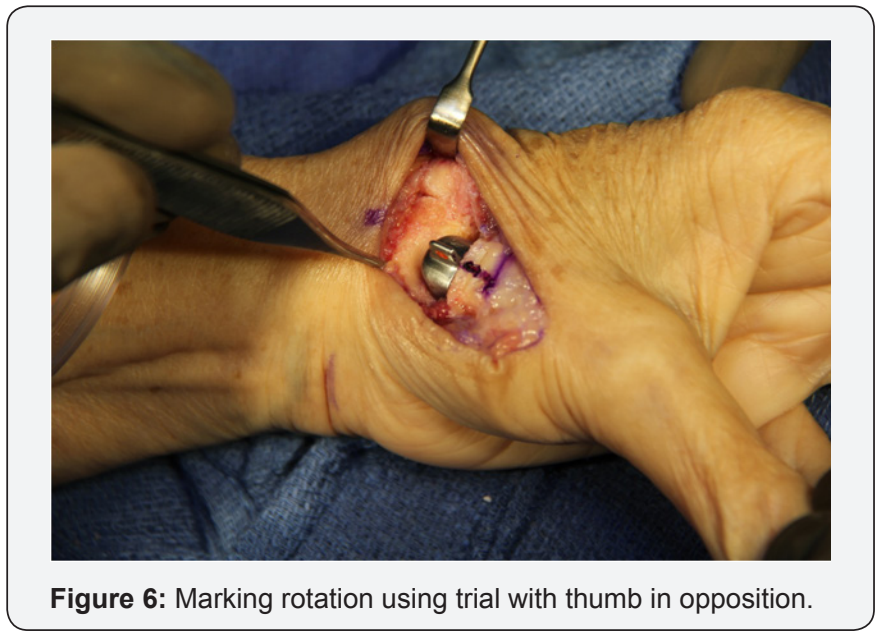

n) Confirm positioning of the implant with fluoroscopy in the PA and lateral planes. Articulate the implant through a full range of motion in both planes with live fluoroscopy to confirm appropriate kinematics and clearance of the volar lip. Remove any offending osteophytes that may impinge and block motion of the implant.

o) The implant provides suture holes for optional suture stabilization in cases with significant instability. Prior to placing the final implant pass a suture through the FCR tendon at the midpoint of the trapezium width using the provided suture passer. Then pass each limb of the suture through the corresponding hole in the selected final implant. This step is optional, as we have found that tethering to the FCR tendon can cause persistent FCR tendonitis and pain. However, it is a useful step if concerned about stability.

p) Select your final implant and align the etched mark on the dorsal side of the implant to the corresponding rotational midpoint created in step 12. Insert the implant until the volar keel engages the cancellous bone of the metacarpal.

q) Use the provided impact or to fully insert the prosthesis. Confirm that the prosthesis is fully seated by manually attempting to remove the implant. The prosthesis should not be able to be removed easily.

r) Once again, place the capsular elevator as a shoehorn over the trapezial articular surface and reduce the final implant. 
Confirm that the FCR tendon is not interposed between the implant and the trapezium. If the optional suture stabilization technique was utilized, tie the two limbs of the suture tightly over the prosethesis (Figure 7).

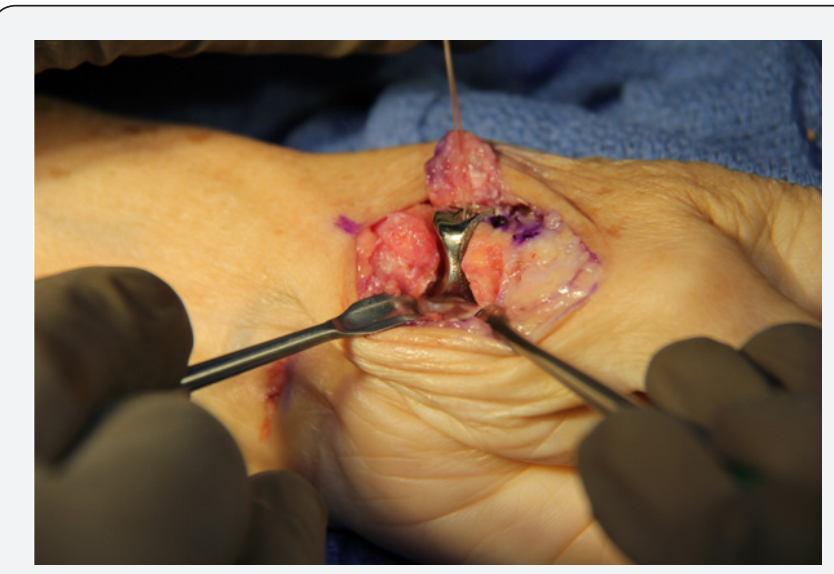

Figure 7: Final implant reduced within the CMC joint.

s) Confirm that the implant has unobstructed motion by placing the joint through a range of motion in radial abduction and opposition. This will confirm the stability of the implant as well as its kinematics. Take final fluoroscopic images of the final implant to document appropriate placement (Figure 8).

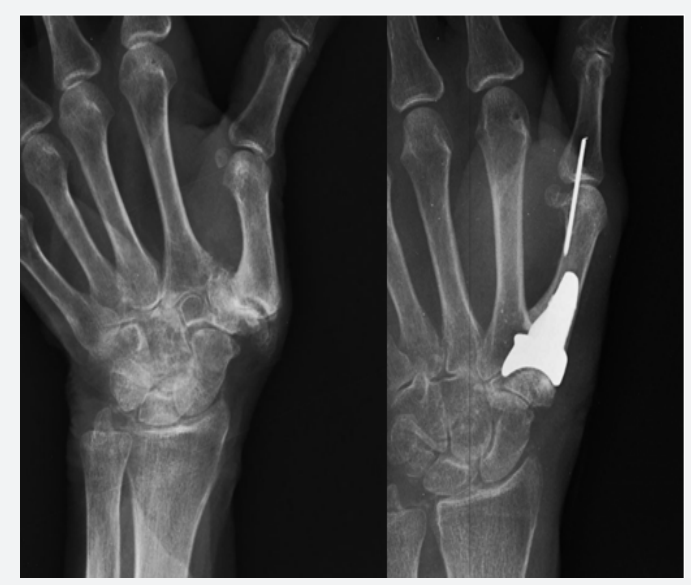

Figure 7: X-Ray images thumb pre-op and post-op with final implant.

t) The capsule is closed over the implant by taking the previously created capsular flap and suturing it to the APL tendon using non-absorbable suture. The skin is then approximated with 4-0 monocryl suture in a subcuticular fashion. Steri-strips are applied to the incision.

u) The thumb is then dressed in a forearm based thumb spica plaster splint for two weeks to provide pain relief until the first post-surgical visit in clinic.

v) Our post-operative protocol is to place the patient into a forearm-based thumb spica cast for 4 weeks to allow the capsule to heal and to continue to minimize pain and swelling.
At the 6-week mark, the patient is placed into a removal handbased thumb controller brace and begins formal hand therapy to regain motion. Strengthening is begun by a certified hand therapist once the surgical pain has resolved. Patients return to work at the 2-month mark once comfortable in the controller brace, if their occupation is clerical in nature or does not require heavy manual labor.

\section{Outcomes}

Since the Stablyx ${ }^{\circledR}$ Arthroplasty System (Skeletal Dynamics, LLC, Miami, FL) was released in 2013, 120 hemiarthroplasty procedures have been performed at the Miami Hand Center, with only 2 revisions. Of these, 12 patients have been followed to the 2-year mark measuring pain, function, strength, range of motion, and trapezial wear [19-22]. Pain was measured on a 10-point visual analog scale (VAS) while resting and during active motion (during a twisting motion/ while unscrewing a bottle cap). At 24 months, the patients' pain was a $0.06 \pm 0.18$ at rest, and $0.34 \pm 0.98$ during active motion. Function and disability was measured using the QuickDASH questionnaire, [23] where 0 is no disability and 100 is total disability.

At 24 months, patients' QuickDASH was a $2.37 \pm 0.86$. Strength was measured as a percentage of the contralateral hand, which might also be diseased. At 24 months, patients' grip strength was $106 \% \pm 26 \%$ and pinch strength was $123 \% \pm 62 \%$ of the contralateral hand. At 24 months, patients had full range of motion, with a Kapandji score of $8.88 \pm 1.25$ out of 10 . Trapezial height was measured using PACSgreen software, with measurements taken from the top to the bottom of the trapezium in the center of the trapezium on a lateral x-ray of the implant. At 24 months, the trapezial height was $99.4 \% \pm 5.4 \%$ of the trapezial height measured at the first post-operative visit (5-7 days postsurgery).

\section{Complications}

The frequently documented complication inthe literature with carpometacarpal implant procedures is loosening of the implant $[14-16,19,20]$. Early total joint prostheses such as the de la Caffiniere prosthesis developed in the 1970's suffered from aseptic loosening resulting in failure rates of 18-44\% [19-21]. With further advancement in implant design and transition to hemiarthroplasty implants, the loosening, subsidence, and subluxation rates have decreased to $1-18 \%$ [14-16]. In the 2012 study performed by Pritchett, hemiarthroplasty of 143 thumbs resulted in zero dislocations and 2 subluxations (1\%) in the series [15]. Woodward followed with zero dislocations in 19 pyrocarbon saddle shaped hemiarthroplasties implanted between 2009 and 2013 [16]. In our series of 12 patients, none had loosening, dislocations, or instability.

Osteonecrosis of the trapezium is a concern as a source of pain and collapse of the thumb in CMC arthritis treated with hemiarthroplasties. Rates of trapezium osteonecrosis is not 
documented in the literature. It is however mentioned as a potential cause of persistent pain that could lead to decreased patient satisfaction $[18,19]$. The two most recently published articles on hemiarthroplasties have one infection combined across both series for a rate of $0.6 \%$ ( 1 of 162 thumbs) and could be attributed to meticulous sterile surgical technique and decreased operative time in comparison to ligament reconstruction and tendon interposition $[15,16]$.

In the event that an implant must be removed, new studies have shown that the outcome of secondary trapeziectomy after failed thumb metacarpal joint replacement arthroplasty is comparable to that of primary trapeziectomy [24,25]. Patients who do not experience the benefits of CMC arthroplasty have reassurance, supported by literature, that they can be successfully revised to trapeziectomy with or without suspensionplasty.

\section{Summary and Discussion}

Basal joint arthritis is common and can encompass 30\% of the patients seen in clinic for debilitating hand pain [1]. Varioussurgical techniques have been devised and documented in the medical literature to approach this problem so that our patients may regain the function they need in their lives. The hemiarthroplasty described above takes a previous technique and refines the surgical approach, as well as the implant, to provide young patients the opportunity to maintain thumb length and function, without sacrificing techniques that have 60 years of proven results.

The surgical dorsal approach with a ulnarly-based capsulotomy that limits proximal release over the trapezium serves to protect the perforating arterial branches that provide blood supply to the trapezium. Minimizing the importance of this fact during surgical dissection could lead to early trapezium osteonecrosis and ultimately failure of the hemiarthroplasty procedure for persistent complaints of pain by the patient. Instability and loosening of previous implants was taken into consideration in the manufacturing of the current implant and the titanium plasma spray, volar keel and longer volar flange has minimized instability in efforts to lower subluxation and dislocation rates. Successfully treated patients with hemiarthroplasty in one limb versus the standard LRTI in the contralateral limb have previously reported that they would undergo the hemiarthroplasty as their first choice between the two procedures if both options were provided [15].

Our current populations of patients who have undergone a previous LRTI have expressed similar sentiments. The hemiarthroplasty also preserves the cosmetic appearance of the thumb for patients as well as the thumb length, a factor proven to improve pinch and grip strength [15-17]. Our current solution of hemiarthroplasty for thumb CMC arthritis demonstrates early patient satisfaction and preserves techniques in the event of implant failure. We would recommend the use of hemiarthroplasty as an alternative to ligament reconstruction tendon interposition in patients with Eaton stage II or Eaton stage III arthritis with good trapezium bone stock with our short-term results.

\section{Conflict of Interest}

Dr. Rubio discloses that he is a consultant for Skeletal Dynamics, LLC.

\section{References}

1. Gillis J, Calder K, Williams J (2011) Review of thumb carpometacarpal arthritis classification, treatment and outcomes. Can J Plast Surg Winter 19(4): 134-138.

2. Neumann DA, Bielefeld T (2013) The carpometacarpal joint of the thumb: stability, deformity, and therapeutic intervention. J Orthop Sports Phys Ther 33(7): 386-399.

3. Rios-Diaz AJ, Metcalfe D, Singh M (2016) Inequities in Specialist Hand Surgeon Distribution across the United States. Plast Reconstr Surg 137(5): 1516-1522.

4. Brunton LM, Wilgis EF (2010) A survey to determine current practice patterns in the surgical treatment of advanced thumb carpometacarpal osteoarthrosis. Hand 5(4): 415-422.

5. Kriegs-Au G, Petje G, Fojtl E (2005) Ligament reconstruction with or without tendon interposition to treat primary thumb carpometacarpal osteoarthritis. Surgical technique. J Bone Joint Surg Am 87 Suppl 1(Pt 1) (Suppl 1): 78-85.

6. Amadio PC, Millender LH, Smith RJ (1982) Silicone spacers or tendon spacer for trapezium resection arthroplasty-comparison of results. J Hand Surg Am 7(3): 237-244.

7. Earp BE (2008) Treatment of advanced CMC joint disease: Trapeziectomy and implant arthroplasty (silastic-metal-synthetic allograft). Hand Clin 24(3): 277-283.

8. Pellegrini VD Jr, Burton RI (1986) Surgical management of basal joint arthritis of the thumb. Part I. Long-term results of silicone implant arthroplasty. J Hand Surg Am 11(3):309-324.

9. Swanson AB, de Groot Swanson G, De Heer DH (1997) Carpal bone titanium implant arthroplasty. 10 years' experience. Clin Orthop Relat Res (342): 46-58.

10. Phaltankar PM, Magnussen PA (2003) Hemiarthroplasty for trapeziometacarpal arthritis - a useful alternative? J Hand Surg $\mathrm{Br}$ 28(1):80-85.

11. Ratner B, Hoffman A, Schoen F, Lemons J (2004) Biomaterials Science: An introduction to materials in medicine, $2^{\text {nd }}$ edition. Academic Press, San Diego, CA, USA.

12. Cook SD, Bechenbaugh RD, Redondo J (1999) Long-term follow-up of pyrolytic carbon metacarpophalangeal implants. J Bone Joint Surg Am 81A: 635-648.

13. Tuttle HG, Stern PJ (2006) Pyrolytic carbon proximal interphalangeal joint resurfacing arthroplasty. J Hand Surg Am 31A:930-939.

14. Martinez de Aragon JS, Moran SL, Rizzo M (2009) Early Outcomes of Pyrolytic Carbon Hemiarthroplasty for the Treatment of TrapezialMetacarpal Arthritis. J Hand Surg Am 34A: 205-212.

15. Pitchett JW, Habryl LS (2012) A promising Thumb Basal Joint Hemiarthroplasty for Treatment of Trapeziometacarpal Osteoarthritis. Clin Orthop Relat Res 470: 2756-2763.

16. Woodward J, Heller J, Jones N (2013) PyroCarbon Implant Hemiarthroplasty for Trapeziometacarpal Arthritis. Tech Hand up Extrem Surg 17(1): 7-12. 
17. Davis DI, Catalano L (2008) Treatment of advanced carpometacarpal joint disease: Carpometacarpal arthroplasty with ligament interposition. Hand Clin 24(3): 263-269.

18. Naidu SH, Kulkarni N, Saunders M (2006) Titanium basal joint arthroplasty: a finite element analysis and clinical study. J Hand Surg Am 31(5):760-765.

19. Ulrich-Vinther M, Puggaard H, Lange B (2008) Prospective 1-Year Follow-up Study Comparing Joint Prosthesis with Tendon Interposition Arthroplasty in Treatment of Trapeziometacarpal Osteoarthritis. J Hand Surg Am 33A: 1369-1377.

20. Barron O, Glickel S, Eaton R (2000) Basal joint arthritis of the thumb. J Am Acad Orthop Surg 8(5): 314-323.

21. Sondergaard L, Konradsen L, Rechnagel K (1991) Long-term followup of the cemented Caffiniere prosthesis for trapezio-metacarpal arthroplasty. J Hand Surg Br 16(4): 428-430.

22. (2017) Florida Orthopaedic Society (FOS) Annual Meeting Conference Proceedings: Early Experience with a Stabilized Saddle Trapeziometacarpal Hemiarthroplasty as presented by Dr. Jorge Orbay on May 21, 2017. The Ocean Reef Club, Key Largo, FL. May 18-21.

23. Hudak PL, Amadio PC, Bombardier C (1996) Development of an upper extremity outcome measure: The DASH (disabilities of the arm, shoulder, and head). Am J Ind Med 29: 602-608.

24. Kaszap B, Daecke W, Jung M (2017) Outcome comparison of primary trapeziectomy versus secondary trapeziectomy following failed total trapeziometacarpal joint replacement. J Hand Surg Am 38(5): 863-871.

25. Cooney WP, Leddy TP, Larson DR (2006) Revision of thumb trapeziometacarpal arthroplasty. J Hand Surg Am 31(2): 219-227.

\section{Your next submission with Juniper Publishers will reach you the below assets}

- Quality Editorial service

- Swift Peer Review

- Reprints availability

- E-prints Service

- Manuscript Podcast for convenient understanding

- Global attainment for your research

- Manuscript accessibility in different formats

( Pdf, E-pub, Full Text, Audio)

- Unceasing customer service

Track the below URL for one-step submission https://juniperpublishers.com/online-submission.php 